\title{
Chronic Pain and Plaque Psoriasis Treated by Lumbar and Stellate Sympathetic Ganglion Block
}

\author{
Sayed Reshad Ghafouri M.D ${ }^{1}$, Muhammad Al-Sabri M.D ${ }^{1}$, Shuaib Ghafoori M.D², Akhtar Ali M.D ${ }^{3}$ \\ ${ }^{1}$ EssenMed House Call Services 2646 E14 $4^{\text {th }}$, Brooklyn, NY 11235; ${ }^{2}$ Shanghai Second Military Medical University 800 Xiangyin Rd, \\ Yangpu Qu, Shanghai, China; ${ }^{3} \mathrm{NY}$ Associated Medical Service, Pain Management 110-04 Jamaica Ave, Richmond Hill, NY 11418.
}

\section{Corresponding Author: \\ Dr. Sayed Reshad Ghafouri \\ Email: sreghafouri@gmail.com}

This is an Open Access article distributed under the terms of the Creative Commons Attribution License (creativecommons.org/ licenses/by/3.0).

Published

February 2, 2019

October 4, 2019

October 25, 2019

\begin{abstract}
Background: Sympathetic ganglion block have been shown to supply immediate relief in various type of chronic pain, but to best of our knowledge, cleaning up of psoriatic lesions after sympathetic ganglion blockage has rarely been reported. Case Report: A 64-year-old patient who has been suffering from chronic pain and plaque psoriasis underwent lumbar and stellate ganglion block in our facility. After the successful treatment, the patient was relieved from pain and psoriatic lesions. Sympathetic ganglion block have been shown to supply immediate relief in various type of chronic pain, but to best of our knowledge, cleaning up of psoriatic lesions after sympathetic ganglion blockage has rarely been reported. Conclusion: We describe a case report in which sympathetic ganglion block successfully treated both chronic pain and plaque psoriasis in a pharmacologically nonresponsive patient.
\end{abstract}

Keywords: Chronic pain, Psoriasis, Stellate Ganglion, Sympathetic Ganglia.

\section{Introduction}

Chronic pain is a persistent pain following its initial onset in the absence of tissue damage with an indeterminate end of healing phase. It is a highly widespread debilitating medical condition which results in poor quality of life and remarkable expenses to the healthcare system [1,2]. Chronic pain has a prevalence of $20-30 \%$ in the general population and affects nearly 100 million of Americans [3]. It is seen more commonly in women, older age and people living in adverse socioeconomic circumstances. Chronic primary pain affects one or more anatomic regions, which sustain longer than three months. It causes functional disability and is believed to be associated with emotional distress [4,5]. An interdisciplinary model of treatment is required to manage and address every component of the patient's pain experience. After a careful assessment, therapy for chronic pain may include medication, nerve blocks, rehabilitation or behavioral interventions and as a less frequently applied therapy, placement of implantable devices to alter the pain can also be included [6].

Anesthetic sympathectomy is an interventional procedure which is indicated for the patients with chronic pain refractory to medical and conservative therapies [7]. Local stellate and lumbar ganglion blocks have been shown to improve pain, function and quality of life in patients with long term and persistent devitalizing pain [8]. We report a case of chronic pain in a patient who was also suffering from plaque psoriasis. The patient was unresponsive to conservative pain management and we decided to experiment lumbar and stellate ganglion blocks which resulted in healing of both chronic pain and plaque psoriasis. 


\section{Case Report}

A 64-year-old Hispanic man was admitted to the chronic pain clinic with a history of 10 years generalized chronic pain. His past medical history included diabetes mellitus type 2 , hypertension and plaque psoriasis covering more than $30 \%$ of his body. He had undergone laparoscopic cholecystectomy ten years before and appendectomy in his twenties. The pain had started spontaneously ten years ago and gradually worsened over time. The patient described the pain as generalized, continuous, intense, mostly involved shoulders, wrists, hands and back. On admission, he rated his pain on average as 10 on a numerical rating scale (NRS). By the time he came to our clinic, he had been on multiple oral analgesics. His treatment regimen for psoriasis lesions were different topical therapies included synthetic vitamin $\mathrm{D}_{3}$ derivatives and Dovonex $0.005 \%$. He had suffered from psoriasis for the past seven years. During that time, he received different therapies including topical and oral steroid and ultraviolet therapy with no improvement.

Physical examination at the time of presentation revealed well-demarcated erythematous scaly annular plaque, scattered on his trunk, face and scalp. His initial workup for chronic pain in different office visits was normal showing no evidence of any defined condition. Given the unsatisfactory outcomes of conservative treatment, we decided to perform a lumbar sympathetic block including mid, upper and lower lumbar ganglions separately and blocked bilateral stellate ganglions. The classic approach for lumbar sympathetic nerve block was applied. We used fluoroscopic guidance and injected a mixture of $1 \%$ lidocaine and $20 \mathrm{mg}$ triamcinolone. At his 8-week following up, the patient appeared to be very satisfied not only because of an excellent relief of his chronic pain, but also reported almost complete resolution of his cutaneous psoriatic lesions. Over the next one year, the patient continued to be free of psoriatic lesions.

\section{Discussion}

Despite outstanding advances, management of chronic pain still remains as an important challenge in the clinical field. Only minority of patients with chronic pain can benefit from pharmacological therapies. In addition, patients with drug therapies often develop drug resistance and experience various side effects where the failure to control pain result in devastating psychosocial, economic and health consequences [9]. So far, different alternative treatments have been developed to relieve the patient's pain. Blocking sympathetic ganglia is one of the alternative treatments which has been experimented for years [10]. It is believed that the inputs from sympathetic ganglia are involved in the pathophysiology of various condition of chronic pain such as loss of regular inhibitory influence on pain and adrenergic hypersensitivity. It is suggested that blocking of the sympathetic neurons interrupts the feedback mechanism in neuronal circuits and diminish neural hyper-excitability. Stellate and lumbar ganglia are part of sympathetic nervous system and their blockage reduce the pain sensitive nerves which eventually reduce the pain $[7,11]$. Single arm and observational studies have shown that $50-90 \%$ of patients whom had undergone therapeutic nerve block had substantial pain relief with no major side effects [12].

The vignette above describes a poorly controlled chronic pain by conservative management which was highly disabling condition and severely degrading the patient's quality of life. Upon admission, the patient was totally symptomatic and suffering from a generalized pain with an intensity of 10 affecting his whole body including shoulders, wrists, hands and back. He was being treated with multiple oral analgesic regimens since his pain had been started, but the treatment was not controlling his pain. The patient was also diagnosed with plaque psoriasis covering more than $30 \%$ of his body for which his dermatologist was following him up in the past seven years. However, 
the implemented treatment was unsuccessful in treating of the lesions. After being submitted to anesthesia and sympathetic ganglia blocks, we observed that nerve blocks significantly improved the pain in the patient. Additionally, the patient reported that his psoriatic lesions were completely cleared up after the procedure and the lesions were not active after a year of follow up.

Recently, few research studies have found that deliberately damaging sensory nerves or preventing the release of specific proteins that play role in skin inflammation by toxins under psoriatic lesions can help to clear up the lesions which results in significant improvement of the psoriasis [13]. Thus, this case report provides the clinical evidence supporting the concept that the psoriasis lesions may be sympathetically mediated which has not been evaluated comprehensively before.

\section{Conclusion}

Sympathetic nerve block has been proven to be an effective interventional treatment in chronic pain for short and long-term remission. Our case demonstrated that and sympathetic ganglion block might be an effective therapy of plaque psoriasis. However, more studies are needed to verify its validity.

Contributors: SRG, MAl-S and SG completed the background research, drafted and edited the manuscript. AA edited and completed the manuscript. SRG will act as a study guarantor. All authors approved the final version of this manuscript. Funding: None; Competing interests: None stated.

\section{References}

1. Weinrib A, Burns L, Mu A, Azam MA, Ladak SJ, McRae $\mathrm{K}$, et al. A case report on the treatment of complex chronic pain and opioid dependence by a multidisciplinary transitional pain service using the ACT Matrix and buprenorphine/naloxone. J Pain Res. 2017;10:747-755.

2. Apkarian AV, Baliki MN, Geha PY. Towards a theory of chronic pain. Prog Neurobiol. 2009;87:81-97.

3. Brekke, M, Hjortdahl, P, Kvien T. Severity of musculoskeletal pain: relationship to socioeconomic inequality. Soc Sci Med. 2002;54:221-228.

4. Treede RD, Rief W, Barke A, Aziz Q, Bennett MI, Benoliel R, et al. A classification of chronic pain for ICD-11. Pain. 2015;156:1003-1007.

5. van Hecke O, Torrance N, Smith BH. Chronic pain epidemiology and its clinical relevance. Br J Anaesth. 2013;111:13-18.

6. Ashburn MA, Staats PS. Management of chronic pain. The Lancet. 1999;353:1865-1869.

7. Menon R, Swanepoel A. Sympathetic blocks. Contin Educ Anaesth Crit Care Pain. 2010;10:88-92.

8. McDonnell JG, Finnerty O, Laffey JG. Stellate ganglion blockade for analgesia following upper limb surgery: Stellate ganglion blockade for postoperative pain. Anaesthesia. 2011;66:611-614.

9. Park HJ, Moon DE. Pharmacologic management of chronic pain. Korean J Pain. 2010;23:99.

10. Ferrillo MG. Treatment of complex regional pain syndrome with stellate ganglion local anesthetic blockade: a case report of one patient's experiences with traditional bupivacaine $\mathrm{HCl}$ and liposome bupivacaine. Clin Case Rep. 2016;4:861-865.

11. Baron R, Jänig W, With H. Sympathetic and afferent neurones projecting into forelimb and trunk nerves and the anatomical organization of the thoracic sympathetic outflow of the rat. J Auton Nerv Syst. 1995;53:205-214.

12. Aslakson R, Cheng J, Vollenweider D, Galusca D, Smith TJ, Pronovost PJ. Evidence-based palliative care in the intensive care unit: A systematic review of interventions. J Palliat Med. 2014;17:219-235.

13. Gilbert E, Ward NL. Efficacy of botulinum neurotoxin type A for treating recalcitrant plaque psoriasis. J Drugs Dermatol. 2014;13:1407-1408. 